\title{
Particle creation in gravitational collapse to a horizonless compact object
}

\author{
Tomohiro Harada, ${ }^{1, *}$ Vitor Cardoso, ${ }^{2,3}$ and Daiki Miyata ${ }^{1}$ \\ ${ }^{1}$ Department of Physics, Rikkyo University, Toshima, Tokyo 171-8501, Japan \\ ${ }^{2}$ CENTRA, Departamento de Física, Instituto Superior Técnico, Universidade de Lisboa, \\ Avenida Rovisco Pais 1, 1049 Lisboa, Portugal \\ ${ }^{3}$ Theoretical Physics Department, CERN 1 Esplanade des Particules, Geneva 23, CH-1211, Switzerland
}

(Received 23 November 2018; published 20 February 2019)

\begin{abstract}
Black holes (BHs) play a central role in physics. However, gathering observational evidence for their existence is a notoriously difficult task. Current strategies to quantify the evidence for BHs all boil down to looking for signs of highly compact, horizonless bodies. Here, we study particle creation by objects which collapse to form ultracompact configurations, with the surface at an areal radius $R=R_{f}$ satisfying $1-\left(2 M / R_{f}\right)=\epsilon^{2} \ll 1$ with $M$ the object mass. We assume that gravitational collapse proceeds in a "standard" manner until $R=R_{f}+2 M \epsilon^{2 \beta}$, where $\beta>0$, and then slows down to form a static object of radius $R_{f}$. In the standard collapsing phase, Hawking-like thermal radiation is emitted, which is as strong as the Hawking radiation of a $\mathrm{BH}$ with the same mass but lasts only for $\sim 40\left(M / M_{\odot}\right)\left[44+\ln \left(10^{-19} / \epsilon\right)\right] \mu \mathrm{s}$. Thereafter, in a very large class of models, there exist two bursts of radiation separated by a very long dormant stage. The first burst occurs at the end of the transient Hawking radiation and is followed by a quiescent stage which lasts for $\sim 6 \times 10^{6}\left(\epsilon / 10^{-19}\right)^{-1}\left(M / M_{\odot}\right)$ yr. Afterwards, the second burst is triggered, after which there is no more particle production and the star is forever dark. In a model with $\beta=1$, both the first and second bursts outpower the transient Hawking radiation by a factor $\sim 10^{38}\left(\epsilon / 10^{-19}\right)^{-2}$.
\end{abstract}

DOI: 10.1103/PhysRevD.99.044039

\section{INTRODUCTION AND SUMMARY}

It is generally accepted that black holes (BHs) can be and have been found in various astrophysical systems, such as $\mathrm{x}$-ray binaries, galactic nuclei, and binary systems sourcing gravitational waves. These systems all contain dark, compact, and massive objects whose properties are all consistent with the $\mathrm{BH}$ paradigm. However, BHs are defined by the existence of an event horizon, which is the boundary of the causal past of future null infinity. By its own definition, finding observational proof for event horizons is impossible [1-4]. Thus, sufficiently compact bodies can mimic BHs at a classical level. Given the crucial role of horizons in a number of fundamental issues, quantifying the evidence for BHs is as important as quantifying, say, the level to which the equivalence principle is satisfied [1-3,5-9].

A natural strategy to test the $\mathrm{BH}$ paradigm is to look for smoking-gun imprints of horizonless bodies. The number of proposals for ultracompact horizonless objects is large and growing (e.g. Refs. [10-12] and see Ref. [2] for a review).

\footnotetext{
*harada@rikkyo.ac.jp
}

Published by the American Physical Society under the terms of the Creative Commons Attribution 4.0 International license. Further distribution of this work must maintain attribution to the author(s) and the published article's title, journal citation, and DOI.
The exterior of such (static) objects is described by the same Schwarzschild geometry as that of a nonspinning BH. Thus, as we stressed already, it is challenging to find evidence of a surface using classical electromagnetic or gravitational waves [1-3,5-9,13-17].

Classical physics predicts measurable differences between ultracompact horizonless stars and $\mathrm{BHs}$, but these may either be inaccessible to observers far away or simply take too long to affect our detectors. However, there is a semiclassical effect which is, seemingly, particular to $\mathrm{BH}$ geometries: Hawking radiation. In fact, when quantum effects are included at a semiclassical level, particles are created and emitted by BHs, and the spectrum of the radiation is thermal, such as that of a black body [18-20]. In Refs. [21-25], quantum particle creation by a collapsing object and its semiclassical effect on the formation of an apparent horizon have been discussed, based on quantum field theory in curved spacetime, in a very general context. Quantum particle creation in horizonless gravitational collapse has also been discussed in the context of naked singularity formation [26-33].

The organization and summary of this work is the following. In Sec. II, we review quantum particle creation in spherically symmetric spacetimes. In Sec. III, we expand on our toy model of a collapsing spacetime by pasting Minkowski and Schwarzschild spacetimes with a timelike shell. In Sec. IV, we review how the present formalism can 
be used to recover a constant particle radiation by $\mathrm{BHs}$, i.e., the Hawking radiation, with an emphasis on transient thermal radiation in the absence of horizon formation. In Sec. V, we introduce a collapse model with a null shell to a horizonless compact object, yielding delta-functional divergent emissions both at the end of the transient Hawking radiation and at the end of the long dormant stage. In Sec. VI, we construct a collapse model with a timelike shell to a horizonless compact object, show the couple of finite bursts of radiation as a common feature in a broad class of models, and present the temporal change of radiation for specific models. Section VII is devoted to discussion. We use units in which $G=c=\hbar=1$.

\section{PARTICLE CREATION IN SPHERICALLY SYMMETRIC SPACETIMES}

Consider a spherically symmetric asymptotically flat spacetime. Let $u$ and $v$ be radial null coordinates, which can be written as $u=t-r$ and $v=t+r$ in the asymptotic region, where $(t, r, \theta, \phi)$ are the usual quasi-Minkowskian spherical coordinates there. Consider a pair of ingoing and outgoing null rays, $v=v_{\text {in }}$ and $u=u_{\text {out }}$, respectively, which are connected at the regular center $r=0$ with each other. The null-ray pairs are depicted in the conformal diagrams for the spacetimes of collapse to a static star and a black hole in Figs. 1(a) and 1(b), respectively. The mapping function $G$ is defined as $v_{\text {in }}=G\left(u_{\text {out }}\right)$. Note that $u$ can be identified with the observer's time at infinity. Following Refs. [21,23], we define

$$
\kappa\left(u_{\text {out }}\right):=-\frac{d}{d u_{\text {out }}} \ln \frac{d v_{\text {in }}}{d u_{\text {out }}}=-\left(\ln G^{\prime}\right)^{\prime}\left(u_{\text {out }}\right),
$$

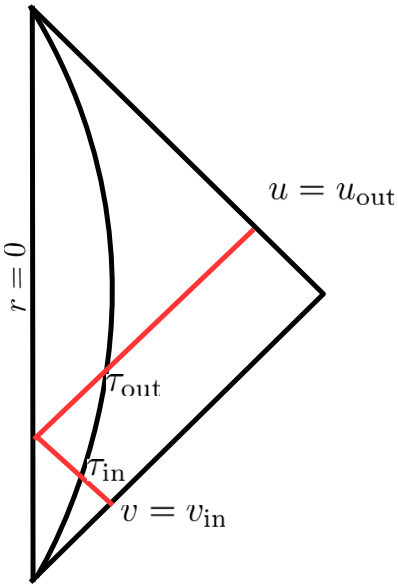

(a)

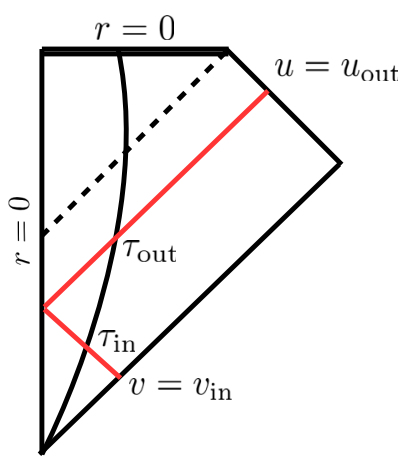

(b)
FIG. 1. The conformal diagrams for the spacetimes of collapse to (a) a static star and (b) a black hole. A pair of outgoing and ingoing null rays with $u=u_{\text {out }}$ and $v=v_{\text {in }}$, respectively, is also depicted in each diagram. which is physically interpreted as the growth rate of redshift of the outgoing photon with respect to the ingoing photon as a function of the retarded time $u_{\text {out }}$.

To calculate quantum radiation, for simplicity, we adopt the same set of assumptions as in Refs. $[18,19,26]$. That is, we assume Gaussian (non-self-interacting) massless scalar fields, adopt the geometrical optics approximation, and take a quantum state containing no particle associated with a mode function $F_{\omega l m}$ which takes the form $F_{\omega l m} \sim$ $(4 \pi \omega)^{-1 / 2} r^{-1} e^{-i \omega v} Y_{l m}(\theta, \phi)$ at past null infinity as an initial quantum state. Then, the function $\kappa(u)$ determines radiation power regularized at future null infinity, through $[20,26,32]$

$$
P_{l m}=\frac{1}{48 \pi}\left(\kappa^{2}+2 \delta \kappa^{\prime}\right),
$$

with $\delta=1$ and 0 for minimally and conformally coupled massless scalars, respectively, for each $(l, m)$ mode. Note that for higher l's, the geometrical optics approximation is not valid and the power is strongly suppressed due to backscattering. Thus, the total power is dominated by sufficiently low $l$ 's. We can thus omit the $(\mathrm{lm})$ subscript in Eq. (2.2) and regard its right-hand side as an order-ofmagnitude estimate of the total power of radiation. The second term in parentheses on the right-hand side of Eq. (2.2) does not contribute to the integrated radiated energy because it is a total derivative; hence, we will mainly concentrate on the first term, i.e., that for the conformally coupled scalar field.

If the function $\kappa(u)$ satisfies the adiabatic condition

$$
\left|\kappa^{\prime}\left(u_{*}\right)\right| \ll \kappa^{2}\left(u_{*}\right),
$$

then the spectrum of outgoing particles at $u=u_{*}$ can be regarded as Planckian with temperature $T$ [21,23],

$$
k T\left(u_{*}\right)=\frac{\kappa\left(u_{*}\right)}{2 \pi},
$$

where $\kappa\left(u_{*}\right)>0$ is assumed.

\section{SPHERICAL SHELL IN VACUUM}

Our model is a spherically symmetric vacuum spacetime with a shell. The areal radius of a timelike shell is given by $r=R(\tau)$, where $\tau$ is the proper time for the observer at rest on the shell. The induced metric on the timelike world tube $\Sigma$ is given by

$$
d s_{\Sigma}^{2}=-d \tau^{2}+R^{2}(\tau) d \Omega^{2},
$$

where $d \Omega^{2}=d \theta^{2}+\sin ^{2} \theta d \phi^{2}$ is the metric on a unit sphere. The interior is described by the Minkowski metric

$$
d s^{2}=-d T^{2}+d r^{2}+r^{2} d \Omega^{2} .
$$

The null coordinates in the interior are $U=T-r$ and $V=T+r$. The exterior is given by the Schwarzschild metric 
$d s^{2}=-\left(1-\frac{2 M}{r}\right) d t^{2}+\left(1-\frac{2 M}{r}\right)^{-1} d r^{2}+r^{2} d \Omega^{2}$.

The standard null coordinates are given by $u=t-r^{*}$ and $v=t+r^{*}$, where $r^{*}:=r+2 M \ln [(r / 2 M)-1]$. The junction condition for the first fundamental form gives $\dot{t}$ and $\dot{T}$, where the dot denotes the derivative with respect to $\tau$. This gives $\dot{U}$ and $\dot{V}$ and $\dot{u}$ and $\dot{v}$. The explicit expressions for them are relegated to Appendix A.

Since $V=V_{\text {in }}$ and $U=U_{\text {out }}$ are related through $V_{\text {in }}=$ $U_{\text {out }}$ at the center $r=0$, we find

$G^{\prime}(u)=\frac{d v_{\text {in }}}{d u_{\text {out }}}=\frac{d v_{\text {in }}}{d \tau_{\text {in }}} \frac{d \tau_{\text {in }}}{d V_{\text {in }}} \frac{d V_{\text {in }}}{d U_{\text {out }}} \frac{d U_{\text {out }}}{d \tau_{\text {out }}} \frac{d \tau_{\text {out }}}{d u_{\text {out }}}=\frac{A_{\text {out }}}{B_{\text {in }}}$,

where

$$
A:=\frac{\dot{U}}{\dot{u}} \quad \text { and } \quad B:=\frac{\dot{V}}{\dot{v}},
$$

$A_{\text {out }}=A\left(\tau_{\text {out }}(u)\right)$, and so on, and $\tau_{\text {out }}(u)$ and $\tau_{\text {in }}(u)$ are the values of $\tau$ when the outgoing and ingoing null rays cross the shell, respectively, as shown in Figs. 1(a) and 1(b). Further, we can obtain the expression for $\kappa(u)$ as follows:

$$
\kappa(u)=C_{\text {out }}-\frac{A_{\text {out }}}{B_{\text {in }}} D_{\text {in }},
$$

where

$$
C:=-\frac{1}{\dot{u}} \frac{d \ln A}{d \tau} \quad \text { and } \quad D:=-\frac{1}{\dot{v}} \frac{d \ln B}{d \tau} .
$$

The first and second terms on the right-hand side of Eq. (3.6) can be regarded as the contributions from the shell at $\tau=\tau_{\text {out }}$ and $\tau=\tau_{\text {in }}$, respectively. We can obtain the explicit expressions for $A, B, C$, and $D$ in terms of $R, \dot{R}$, and $\ddot{R}$, which are relegated to Appendix A.

For reference, if the shell is marginally bound and made of dust, then the junction condition for the second fundamental form gives

$\dot{R}^{2}=-1+\left(1+\frac{M}{2 R}\right)^{2} \quad$ and $\quad \ddot{R}=-\frac{M}{2 R^{2}}\left(1+\frac{M}{2 R}\right)$.

However, we will not assume any equation of state for the surface energy density and pressure on the shell. Instead, we specify the dynamics of the shell. The evolution of the surface energy density and pressure will then be determined by the junction condition for the second fundamental form. This freedom has a price: our simplistic model may contain unphysical matter content with an exotic equation of state. We should stress that our purpose here is not to produce alternatives to $\mathrm{BHs}$; rather, we are interested in understanding possible consequences of failing to produce horizons. This program, if successful, then allows us to quantify in a better way the evidence for $\mathrm{BHs}$ and to strengthen the $\mathrm{BH}$ paradigm.

\section{PARTICLE CREATION IN STANDARD-COLLAPSE PHASE}

Conventionally, to derive the Hawking radiation, the expandability of $R(\tau)$ with respect to $\tau$ at the entry into the horizon $\tau=\tau_{H}$ has often been assumed [20]. However, such an assumption seems to prescribe the behavior of the shell at an event which is not in the causal past of the observer. Here we show that the expandability at $\tau=\tau_{H}$ is unnecessary and, hence, that the (temporarily) thermal radiation does not need any horizon.

Instead, observing the dust-shell collapse described by Eq. (3.8), we assume that the standard collapse is divided into the following two phases:

(i) Phase 0 , an early-collapse phase: $\tau<\tau_{0}$ or $R>4 M$. We assume

$$
1-\frac{2 M}{R}>\frac{1}{2}, \quad|\dot{R}| \lesssim 1, \quad \text { and } \quad|\ddot{R}| \lesssim \frac{1}{2 M} .
$$

We can additionally assume that the shell is initially static at some radius $R_{i}$.

(ii) Phase 1, a late-collapse phase: $\tau>\tau_{0}$ or $2 M<$ $R<4 M$. We assume

$$
\begin{gathered}
1-\frac{2 M}{R}<\frac{1}{2}, \quad 1-\frac{2 M}{R}<\dot{R}^{2}, \quad \dot{R}=O(1), \\
\text { and } \ddot{R}=O\left((2 M)^{-1}\right) .
\end{gathered}
$$

The functions $A, B, C$, and $D$ take expressions $A_{j}, B_{j}, C_{j}$, and $D_{j}$ for phase $j$. The explicit expressions are relegated to Appendix B. The transition between the above two regimes occurs at $\tau=\tau_{0}$ when $R=4 M$. This scenario of standard collapse is then consistent with the dust-shell collapse.

Denoting the outgoing null ray in the Schwarzschild region which leaves the shell at $\tau=\tau_{0}$ with $u=u_{0}$, we can obtain the expression for $G^{\prime}(u)$ and $\kappa(u)$ separately for $u<u_{0}$ and $u>u_{0}$. To do this, it is a key to determine when the outgoing null ray crosses the shell outwardly and when the ingoing null ray, which is a counterpart of the outgoing null ray in the pair, crosses the shell inwardly. If the outgoing null ray crosses the shell outwardly in phase $i$ at $\tau=\tau_{\text {out }}$ and the ingoing null ray crosses the shell inwardly in phase $j$ at $\tau=\tau_{\text {in }}$, we classify the null-ray pair as $(i, j)$. For the null-ray pair of class $(i, j), G^{\prime}$ and $\kappa$ are given by 


$$
\begin{aligned}
G^{\prime}(u) & =\frac{A_{i, \text { out }}(u)}{B_{j, \text { in }}(u)} \text { and } \\
\kappa(u) & =C_{i, \text { out }}(u)-\frac{A_{i, \text { out }}(u)}{B_{j, \text { in }}(u)} D_{j, \text { in }}(u),
\end{aligned}
$$

respectively, where we use the notation $A_{i, \text { out }}(u)=$ $A_{i}\left(\tau_{\text {out }}(u)\right)$ and so on. Then, we can find that there are two radiation stages.

(i) $u<u_{0}$ : Since $\tau_{\text {in }}<\tau_{\text {out }}<\tau_{0}$, the null-ray pairs are classified as $(0,0)$. Using Eqs. (4.3) and (B1), we have

$$
\kappa(u) \simeq-\left[\frac{M}{R}\left(\ddot{R}-\frac{|\dot{R}|}{R}\right)\right]_{\text {out }}-\left[\frac{M}{R}\left(\ddot{R}+\frac{|\dot{R}|}{R}\right)\right]_{\text {in }} .
$$

Therefore, we can conclude that $|\kappa| \lesssim 1 /(4 M)$. Thus, the radiation for $u<u_{0}$, which may be called pre-Hawking radiation, is weaker than the standard Hawking radiation.

(ii) $u>u_{0}$ : For $\tau_{\text {in }}<\tau_{0}$, the null-ray pairs are classified as $(1,0)$, while, for $\tau_{\text {in }}>\tau_{0}$, the null-ray pairs are classified as (1,1). For both cases, from Eqs. (4.3), (B1), and (B8) we have the same expression for $\kappa(u)$ :

$$
\kappa(u) \simeq C_{\mathrm{out}} \simeq \frac{1}{4 M}
$$

Here, we discuss the radiation for $u>u_{0}$. Using Eq. (2.2), we obtain

$$
P \simeq P_{H}=\frac{1}{48 \pi} \frac{1}{16 M^{2}} .
$$

This is the reproduction of the Hawking radiation. Since the first term is dominant in the expression for $\kappa(u)$ in Eq. (4.3), the Hawking radiation (whether transient or eternal) originates from the behavior of the shell in the late-collapse phase at $\tau=\tau_{\text {out }}$.

Equations (2.1) and (2.4) give temporarily thermal radiation with temperature

$$
k T\left(u_{*}\right) \simeq k T_{H}=\frac{1}{8 \pi M},
$$

where we can easily see that the adiabatic condition (2.3) is also satisfied. Since no horizon has formed yet, this means that transient Hawking radiation does not need any horizon. If the late-collapse phase continues up until $R \simeq 2 M\left(1+\epsilon^{2}\right)$, then the transient Hawking radiation arises and lasts for $\Delta u \simeq 4 M \ln \epsilon^{-2}$, which can be seen from Eq. (B7). Therefore, the radiated energy through this transient Hawking radiation is given by

$$
E \simeq \frac{1}{48 \pi} \frac{\ln \epsilon^{-2}}{4 M}
$$

In the limit $\epsilon \rightarrow 0$, which may correspond to the formation of an event horizon depicted in Fig. 1(b), the Hawking radiation continues eternally and the energy radiated goes to infinity.

\section{COLLAPSE TO AN ULTRACOMPACT OBJECT WITH A NULL SHELL}

We now review and reanalyze an exact collapse model with a null shell, which can result in a static compact star with radius slightly larger than $2 M$. The schematic diagram of this model - introduced in Ref. [34]—is shown in Fig. 2 and consists of three phases. Note that these phases are different from those in the timelike-shell model.

(i) $u<u_{0}$ : Initially, the shell is static with $R=R_{i}$.

(ii) $u_{0}<u<u_{1}$ : At $u=u_{0}$, the shell suddenly turns ingoing null with $V=0$. Since the shell is also given by $v=$ const, we find

$$
\begin{aligned}
u-u_{0}= & U-4 M \ln \left(\frac{-U}{4 M}-1\right)+2 R_{i} \\
& +4 M \ln \left(\frac{R_{i}}{2 M}-1\right),
\end{aligned}
$$

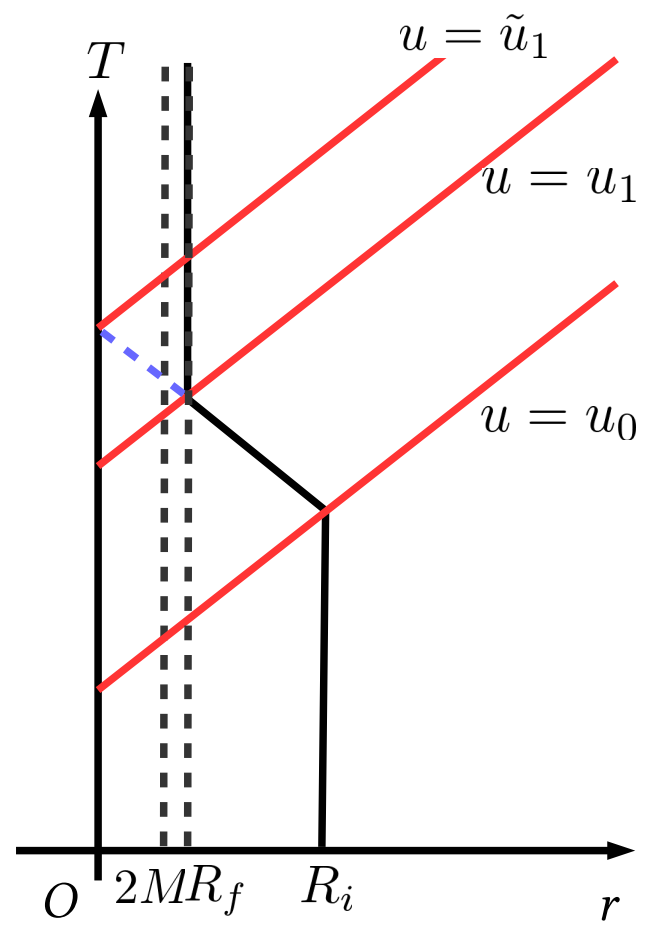

FIG. 2. The collapse model with an ingoing null shell. The static shell at $R=R_{i}$ changes to an ingoing null shell at $u=u_{0}$ and again becomes static with $R=R_{f}$ at $u=u_{1}$. The ingoing null shell is extended to the Minkowski region with an ingoing null ray, which is denoted by a blue dashed line, and reflected to an outgoing null ray that passes the shell outwardly to the Schwarzschild region, which is denoted with a red line labeled $u=\tilde{u}_{1}$. This model was introduced in Ref. [34]. 
where $U=-2 R_{\text {out }}$ is a monotonically increasing function of $u$ from $-2 R_{i}$ to $-2 R_{f}$.

(iii) $u>u_{1}$ : When the shell reaches the radius $R_{f}:=$ $2 M /\left(1-\epsilon^{2}\right)$ at $u=u_{1}$, it stops and becomes static again, where $u_{1}$ is determined by

$$
\begin{aligned}
u_{1}-u_{0}= & -2\left[\frac{2 M}{1-\epsilon^{2}}+2 M \ln \frac{\epsilon^{2}}{1-\epsilon^{2}}\right] \\
& +2\left[R_{i}+2 M \ln \left(\frac{R_{i}}{2 M}-1\right)\right] .
\end{aligned}
$$

We treat $\epsilon$ as a constant free parameter satisfying $0<\epsilon<1$.

We also define $\tilde{u}_{1}$ such that the ingoing null shell $V=0$ is extended with an ingoing null ray to the center $r=0$ in the Minkowski region, being reflected to the outgoing null ray and going through the shell to an outgoing null ray $u=\tilde{u}_{1}$ in the Schwarzschild region. We can find

$$
\tilde{u}_{1}=u_{1}+\frac{4 M}{\epsilon\left(1-\epsilon^{2}\right)} .
$$

The functions $G^{\prime}(u)$ and $\kappa(u)$ are calculated as follows:

(i) $u<u_{0}$ : All null-ray pairs are classified as $(0,0)$, for which we have $G^{\prime}(u)=1$ and $\kappa(u)=0$.

(ii) $u_{0}<u<u_{1}$ : All null-ray pairs are classified as $(1,0)$, for which

$$
\begin{aligned}
G^{\prime}(u) & =\left(1-\frac{2 M}{R_{i}}\right)^{-1 / 2}\left(1+\frac{4 M}{U}\right), \\
\kappa(u) & =-\frac{G^{\prime \prime}}{G^{\prime}}=\frac{4 M}{U^{2}},
\end{aligned}
$$

where $U(u)$ is implicitly given by Eq. (5.1).

(iii) $u_{1}<u<\tilde{u}_{1}$ : All null-ray pairs are classified as $(2,0)$, for which

$$
G^{\prime}(u)=\epsilon\left(1-\frac{2 M}{R_{i}}\right)^{-1 / 2}, \quad \kappa(u)=0 .
$$

(iv) $u>\tilde{u}_{1}$ : All null-ray pairs are classified as $(2,2)$, for which we have $G^{\prime}(u)=1$ and $\kappa(u)=0$.

Therefore, particles are emitted for $u_{0}<u<u_{1}$, but not for $u<u_{0}, u_{1}<u<\tilde{u}_{1}$, and $\tilde{u}_{1}<u$. For $\epsilon \ll 1$, the radiation for $u_{0}<u<u_{1}$ can be regarded as temporarily thermal with temperature $k T=\kappa(u) /(2 \pi)=M /\left(2 \pi R_{\text {out }}^{2}\right)$. Therefore, $k T \simeq 1 /(8 \pi M)$ for $1-2 M / R_{\text {out }} \ll 1$. This is transient Hawking radiation.

In this model, we have bursts of radiation at $u=u_{0}, u_{1}$, and $\tilde{u}_{1}$ because $G^{\prime}$ changes discontinuously then and $\kappa$ is given by Eq. (2.1). The discontinuities in $\left(-\ln G^{\prime}\right)$, which we denote with $\Delta\left(-\ln G^{\prime}\right)$, are given as follows:

$$
\Delta\left(-\ln G^{\prime}\right)_{u=u_{0}}=\ln \left(1-\frac{2 M}{R_{i}}\right)^{-1 / 2},
$$

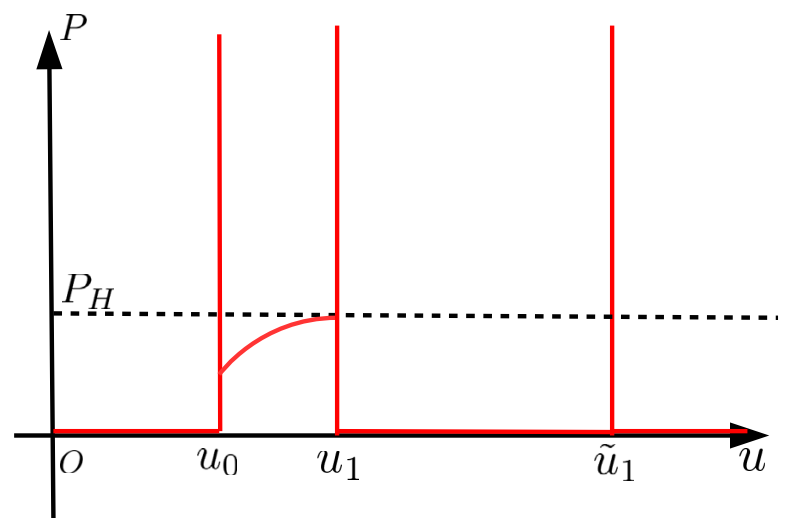

FIG. 3. The schematic figure of the evolution of the power in the collapse to a highly compact object in the null-shell model introduced in Ref. [34]. The three vertical lines denote deltafunctional divergences at $u=u_{0}, u_{1}$, and $\tilde{u}_{1}$, while there appears transient Hawking radiation for $u_{0}<u<u_{1}$.

$$
\begin{gathered}
\Delta\left(-\ln G^{\prime}\right)_{u=u_{1}}=-\ln \epsilon^{-1}, \\
\Delta\left(-\ln G^{\prime}\right)_{u=\tilde{u}_{1}}=-\ln \epsilon^{-1}+\ln \left(1-\frac{2 M}{R_{i}}\right)^{1 / 2} .
\end{gathered}
$$

More precisely, the bursts are described by the square of a delta function at $u=u_{0}, u_{1}$, and $\tilde{u}_{1}$, which suggests infinite radiated energy in an infinitesimal span of time. We schematically plot the evolution of the power of radiation in Fig. 3. The discontinuity in $\left(-\ln G^{\prime}\right)$ is positive and $O(1)$ at $u=u_{0}$, while it is negative and $O\left(\ln \epsilon^{-1}\right)$ at both $u=u_{1}$ and $u=\tilde{u}_{1}$ for $\epsilon \ll 1$. [This divergent behavior was overlooked in Ref. [34]. For example, in Fig. 2 of Ref. [34], there should be three vertical lines indicating delta-functional divergences at $u /(2 M)=u_{0} /(2 M)=0, u_{1} /(2 M) \simeq 19.49$, and $\tilde{u}_{1} /(2 M) \simeq 29.91$ for the choice $u_{0}=0, R_{i}=12 M$, and $\epsilon=0.2$.] The delta-functional burst at $u=u_{0}$ can be removed if the onset of the collapse process is adiabatic. On the other hand, the bursts at $u=u_{1}$ and $\tilde{u}_{1}$ are of more physical interest.

\section{COLLAPSE TO AN ULTRACOMPACT OBJECT WITH A TIMELIKE SHELL}

\section{A. Phases of the shell dynamics}

The features discussed in the null-shell model (in particular the bursts of radiation both at the end of transient Hawking radiation and at the end of the long dormant stage) are of physical interest. However, the deltafunctional divergence is clearly unphysical and arises from the instantaneous transitions from the static shell to null at $u=u_{0}$ and the null shell to timelike at $u=u_{1}$. We also see that the power emitted is finite, as long as $\ddot{R}$ and $\dot{R}$ are finite [cf. Eq. (3.6) and Appendix A].

To have a smooth process and extract meaningful physics, we propose a collapse model of a timelike shell 
with finite $\ddot{R}$ and $\dot{R}$. This model consists of five phases: an early-collapse phase, late-collapse phase, early-braking phase, late-braking phase, and final static phase.

(i) Phase 0, an early-collapse phase: $\tau<\tau_{0}$ or $R>4 M$. This phase is identical to that in standard collapse discussed in Sec. IV; in particular we assume that $1-2 M / R>1 / 2,|\dot{R}| \lesssim 1$, and $|\ddot{R}| \lesssim 1 /(2 M)$.

(ii) Phase 1, a late-collapse phase: $\tau_{0}<\tau<\tau_{1}$ or $R_{b}<R<4 M$. This phase is also identical to that in standard collapse discussed in Sec. IV, i.e., $1-2 M / R<1 / 2,1-2 M / R<\dot{R}^{2}, \dot{R}=O(1)$, and $\ddot{R}=O\left((2 M)^{-1}\right)$.

(iii) Phase 2, an early-braking phase: $\tau_{1}<\tau<\tau_{2}$ or $R_{2}<R<R_{b}$. We assume that at $\tau=\tau_{1}$ or $R=R_{b}$, the shell begins to brake. For $\tau_{1}<\tau<\tau_{2}$, we assume the following inequality:

$$
1-\frac{2 M}{R}<\dot{R}^{2}
$$

(iv) Phase 3, a late-braking phase: $\tau_{2}<\tau<\tau_{3}$ or $R_{f}<R<R_{2}$. We assume that at $\tau=\tau_{2}$, when $R=R_{2}$, the following equality holds:

$$
1-\frac{2 M}{R}=\dot{R}^{2} .
$$

For $\tau_{2}<\tau<\tau_{3}$, the following inequality holds:

$$
1-\frac{2 M}{R}>\dot{R}^{2}
$$

The radius of the shell approaches the final value $R_{f}$.

(v) Phase 4, a final static phase: $\tau>\tau_{3}$ or $R=R_{f}$. We assume that the shell smoothly stops at $\tau=\tau_{3}$, when $R=R_{f}=2 M /\left(1-\epsilon^{2}\right)$. Later on, the shell is completely static.

For later convenience, as is seen in Fig. 4, we label as $u=u_{0}, u_{1}, u_{2}$, and $u_{3}$ those outgoing null rays in the Schwarzschild region which leave the shell outwardly at $\tau=\tau_{0}, \tau_{1}, \tau_{2}$, and $\tau_{3}$, respectively. We use $u=\tilde{u}_{1}, \tilde{u}_{2}, \tilde{u}_{3}$ for those outgoing null rays which are traced back through the center to ingoing null rays and reach the shell at $\tau=\tau_{1}$, $\tau_{2}, \tau_{3}$, respectively. We denote that the proper times when the outgoing null rays $u=\tilde{u}_{1}, \tilde{u}_{2}, \tilde{u}_{3}$ cross the shell outwardly as $\tau=\tilde{\tau}_{1}, \tilde{\tau}_{2}, \tilde{\tau}_{3}$, respectively.

\section{B. Post-Hawking burst}

We find that the emission of bursts of radiation both at the end of the transient Hawking radiation and at the end of a long dormant stage is a general feature of quantum particle creation in setups leading to a compact horizonless object. Here, we briefly describe this phenomenon.

For $u_{1}<u<u_{3}$, the observer receives the outgoing null ray which left the shell outwardly in the braking phase, and which can be traced back to the ingoing null ray which

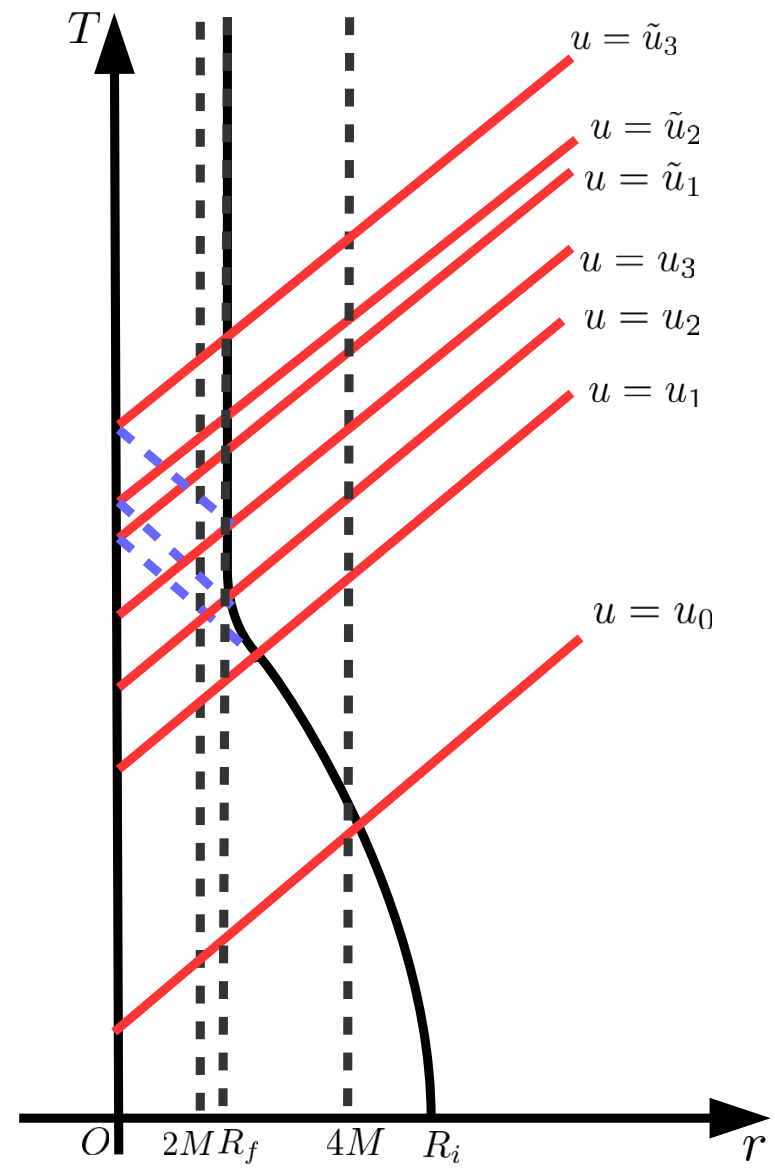

FIG. 4. The collapse model with a timelike shell. The shell enters $R=4 M$ at $\tau=\tau_{0}$, begins to brake at $\tau=\tau_{1}$, and stops at $\tau=\tau_{3}$. Between $\tau_{1}$ and $\tau_{3}$, there is a moment $\tau_{2}$, when the equality $1-2 M / R=\dot{R}^{2}$ is satisfied. The outgoing null rays which pass the shell outwardly to the Schwarzschild region at $\tau=\tau_{0}, \tau_{1}, \tau_{2}$, and $\tau_{3}$ are denoted by red lines labeled $u=u_{0}, u_{1}$, $u_{2}$, and $u_{3}$, respectively. The ingoing null rays denoted by blue dashed lines leave the shell at $\tau=\tau_{1}, \tau_{2}$, and $\tau_{3}$, and are reflected to outgoing null rays passing the shell outwardly to the Schwarzschild region denoted by red lines labeled $u=\tilde{u}_{1}, \tilde{u}_{2}$, and $\tilde{u}_{3}$, respectively.

crosses the shell inwardly in the standard-collapse phase. From Appendix B, $\kappa(u)$ is estimated as

$$
\begin{aligned}
\kappa(u) & =C_{2, \text { out }}+O\left(\epsilon(2 M)^{-1}\right) \\
& \simeq-\left[\frac{\ddot{R}}{2 \dot{R}^{2}}\left(1-\frac{2 M}{R}\right)\left(1-\frac{|\dot{R}|}{\sqrt{1+\dot{R}^{2}}}\right)\right]_{\text {out }}+\frac{1}{4 M}, \\
\kappa(u) & =C_{3, \text { out }}+O\left(\epsilon(2 M)^{-1}\right) \simeq-\ddot{R}_{\text {out }}+\left[\frac{|\dot{R}|}{4 M \sqrt{1-\frac{2 M}{R}}}\right]_{\text {out }},
\end{aligned}
$$

for $u_{1}<u<u_{2}$ and $u_{2}<u<u_{3}$, respectively. Note that the factor $\left(1-\frac{2 M}{R}\right) / \dot{R}^{2}$ is generally an increasing function 
for $u_{1}<u<u_{3}$, which is much smaller than unity at $u=u_{1}$, unity at $u=u_{2}$, and diverging at $u=u_{3}$. In the above expressions, the second term can be regarded as the transient Hawking radiation, which keeps constant for $u_{1}<$ $u<u_{2}$ and decays for $u_{2}<u<u_{3}$. This implies that $u_{2}$ (or $\tau_{2}$ ) plays a clear physical role: it triggers the decay of the transient Hawking radiation. On the other hand, the first term is negative and dominates the second term if $\ddot{R} \gtrsim$ $1 /(2 M)$ for $u_{2} \lesssim u<u_{3}$. The emission due to the first term completely ends at $u=u_{3}$. This gives a burst of radiation at the end of the transient Hawking radiation around $u=u_{2}$, which we call a post-Hawking burst. This particle creation occurs due to the braking of the shell at $\tau=\tau_{\text {out }}$. The details of the burst depend on the specific behavior of the shell in the braking phase.

\section{Late-time burst from a static star}

Next, we consider the interval $\tilde{u}_{1}<u<\tilde{u}_{3}$, when the ingoing null ray crosses the shell inwardly in the braking phase and the outgoing null ray crosses the shell outwardly in the final static phase. In this case, from Appendix B, $\kappa$ is negative and estimated as

$$
\begin{gathered}
\kappa(u)=-\epsilon \frac{D_{2, \mathrm{in}}}{B_{2, \mathrm{in}}} \simeq-\epsilon\left[\frac{\ddot{R}}{|\dot{R}| \sqrt{1+\dot{R}^{2}}}\right]_{\mathrm{in}} \\
-\epsilon\left[\frac{1}{2|\dot{R}|\left(\sqrt{1+\dot{R}^{2}}-|\dot{R}|\right)}\right]_{\mathrm{in}} \frac{1}{4 M}, \\
\kappa(u)=-\epsilon \frac{D_{3, \mathrm{in}}}{B_{3, \text { in }}} \simeq-\epsilon\left[\frac{\ddot{R}}{\sqrt{1-\frac{2 M}{R}}}\right]_{\mathrm{in}}-\epsilon\left[\frac{|\dot{R}|}{1-\frac{2 M}{R}}\right]_{\mathrm{in}} \frac{1}{4 M},
\end{gathered}
$$

for $\tilde{u}_{1}<u<\tilde{u}_{2}$ and $\tilde{u}_{2}<u<\tilde{u}_{3}$, respectively. Therefore, if $\ddot{R} \gtrsim 1 /(2 M)$ at $\tau=\tau_{2}$ in the braking phase, the first term in the above expressions dominates $\kappa(u)$ at $u=\tilde{u}_{2}$, and, hence, particle creation occurs due to the braking at $\tau=\tau_{\text {in }}$. This may be regarded as the ingoing part of the postHawking burst propagating through the center and becoming an outgoing flux. Even if $\ddot{R}$ is totally negligible in the braking phase, the second term in the above expressions describe a burst of radiation with a peak $\kappa \simeq-1 /(4 M)$ at $u=\tilde{u}_{2}$. This may be regarded as the ingoing part of the transient Hawking radiation propagating through the center and becoming an outgoing flux.

Whether the deceleration is effective in particle creation or not, the observation of the burst is delayed from the direct observation of the deceleration at $u=u_{2}$ by $\tilde{u}_{2}-$ $u_{2} \simeq 4 M / \epsilon$.

\section{Time dependence of particle creation}

We will discuss the whole temporal change of radiation for specific models below.

\section{Model A: Exponentially slowed-down model}

First, we assume that $R-R_{f} \propto e^{-\sigma \tau}$ for $\tau_{1}<\tau<\tau_{3}^{\prime}$ except for the short interval $\tau_{3}^{\prime}<\tau<\tau_{3}$, when $R$ smoothly settles down to the final fixed radius $R_{f}$ at $\tau=\tau_{3}$, by introducing the deceleration parameter $\sigma$ such that $\ddot{R}=$ $\sigma|\dot{R}|=\sigma^{2}\left(R-R_{f}\right)$ with

$$
\sigma=\frac{\left|\dot{R}_{b}\right|}{R_{b}-R_{f}}
$$

where $\left|\dot{R}_{b}\right|=O(1)$. We parametrize $R_{b}$ through $R_{b}-R_{f}=$ $2 M \epsilon^{2 \beta}$. For $\beta=1 / 2$, we have

$$
1-\frac{2 M}{R} \simeq\left\{\begin{array}{cc}
\epsilon e^{-\sigma\left(\tau-\tau_{1}\right)} & \left(\tau_{1}<\tau<\tau_{2}\right) \\
\epsilon^{2} & \left(\tau_{2}<\tau<\tau_{3}\right)
\end{array},\right.
$$

while for $\beta \geq 1$, we have

$$
1-\frac{2 M}{R} \simeq \epsilon^{2}
$$

for $\tau_{1}<\tau<\tau_{3}$. We assume that $\tau_{3}-\tau_{2} \simeq \sigma^{-1}$ for simplicity. See Appendix C for the estimate of $\tau_{2}$.

Assuming $\beta \geq 1 / 2$ for simplicity, for the post-Hawking burst, $\kappa(u)$ peaks at $u=u_{2}$ with

$$
\kappa \simeq-\epsilon \sigma \simeq-\frac{1}{2 M \epsilon^{2 \beta-1}} .
$$

The peak power and energy radiated in the post-Hawking burst are roughly estimated to

$$
P \simeq \epsilon^{-2(2 \beta-1)} P_{H} \quad \text { and } \quad E \simeq \epsilon \sigma \sim \frac{1}{2 M \epsilon^{2 \beta-1}} .
$$

So, if $\beta>1 / 2$, the power and the energy radiated in the post-Hawking burst dominate those of the transient Hawking radiation.

It is interesting to look into the late-time burst. We can find $\kappa(u)$ is nearly constant with

$$
\kappa(u) \simeq-\epsilon \sigma \simeq-\frac{1}{2 M \epsilon^{2 \beta-1}}
$$

for $\tilde{u}_{1}<\tilde{u}<\tilde{u}_{2}$ and decays to zero for $\tilde{u}_{2}<u<\tilde{u}_{3}$. The peak power is as strong as the Hawking radiation for $\beta=1 / 2$ and is stronger for $\beta>1 / 2$. For $\tilde{u}_{1}<u<\tilde{u}_{2}$, we can see

$$
\left|\kappa^{\prime}\right| \sim \frac{\epsilon \sigma}{\sigma^{-1} \epsilon^{-1}\left(\ln \epsilon^{-1}\right)} \sim \frac{\kappa^{2}}{\ln \epsilon^{-1}} \ll \kappa^{2},
$$

where we have used Eq. (C8). Therefore, the late-time burst can be regarded as adiabatic. However, this cannot be interpreted as a Planckian distribution with negative temperature: the stationary phase approximation or saddle point 


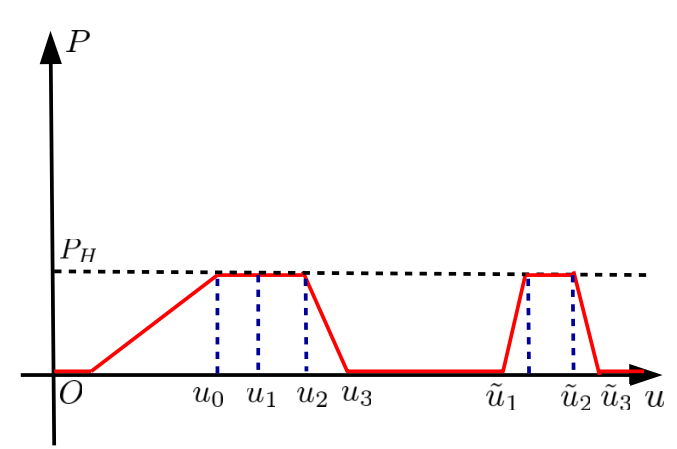

(a) $\beta=1 / 2$

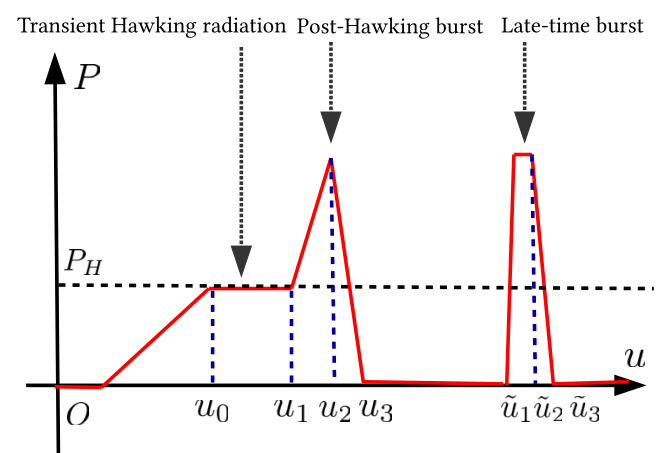

(b) $\beta=1$

FIG. 5. The schematic time dependence of radiation emitted in the timelike collapse to a highly compact object in model A, in which the shell is exponentially slowed down in the braking phase. The shell begins braking at $R=R_{b}=R_{f}+2 M \epsilon^{2 \beta}$ for (a) $\beta=1 / 2$ and (b) $\beta=1$. We here neglect the power of the order of $\epsilon^{2} P_{H}$.

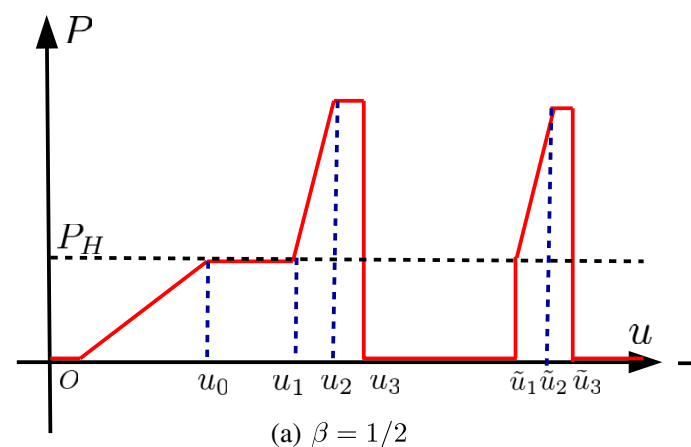

(a) $\beta=1 / 2$

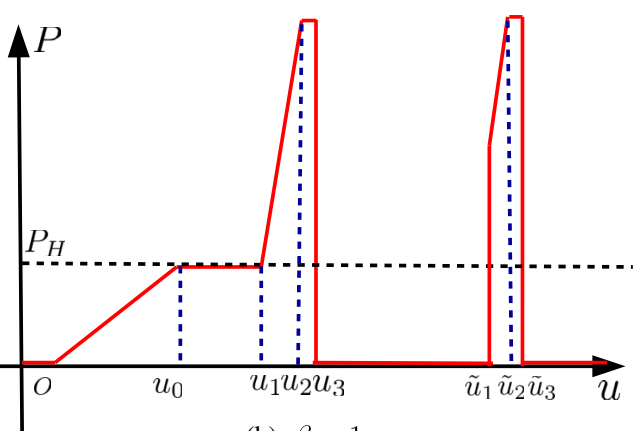

(b) $\beta=1$

FIG. 6. Same as Fig. 5 but in model B, in which the deceleration is constant in the braking phase.

approximation to derive the Planck distribution [21,23] is simply not applicable. ${ }^{1}$ The radiated energy during the burst is calculated to

$$
E \simeq \frac{1}{48 \pi} \sigma \epsilon \ln \epsilon^{-1} \simeq \frac{1}{48 \pi} \frac{\ln \epsilon^{-1}}{2 M \epsilon^{2 \beta-1}} .
$$

For $\beta=1 / 2$, this is approximately equal to energy radiated through the transient Hawking radiation, while for $\beta>1 / 2$, this dominates the latter. The temporal dependences of particle emission are summarized for $\beta=1 / 2$ and $\beta=1$ in Fig. 5.

\section{Model B: Constant-deceleration model}

Next we consider a technically simpler model, where the deceleration $a$ of the shell is constant for $\tau_{1}<\tau<\tau_{3}$ with

$$
a=\frac{\dot{R}_{b}^{2}}{2\left(R_{b}-R_{f}\right)},
$$

where $\left|\dot{R}_{b}\right|=O(1)$. We can naturally assume $a \gg 1 /(4 M)$. Therefore, $\tilde{u}_{3}-\tilde{u}_{1} \sim \epsilon^{-1} a^{-1}$ and $\tilde{u}_{3}-\tilde{u}_{2} \sim a^{-1}$ as derived in Appendix C. We parametrize $R_{b}-R_{f}=2 M \epsilon^{2 \beta}(\beta>0)$ as in the previous model.

\footnotetext{
${ }^{1}$ The authors are grateful to S. Kinoshita for highlighting this issue.
}

For the post-Hawking burst, $\kappa$ decreases from $O\left((2 M)^{-1}\right)$ to $-1 /(4 M) \epsilon^{-2 \beta}$ for $u_{1}<u<u_{2}$ and keeps constant with $\kappa \simeq-1 /(4 M) \epsilon^{-2 \beta}$ for $u_{2}<u<u_{3}$. For the late-time burst, $\kappa$ decreases from $-1 /(4 M) \epsilon^{-(2 \beta-1)}$ to $-1 /(4 M) \epsilon^{-2 \beta}$ for $\tilde{u}_{1}<$ $u<\tilde{u}_{2}$ and keeps constant with $\kappa \simeq-1 /(4 M) \epsilon^{-2 \beta}$ for $\tilde{u}_{2}<u<\tilde{u}_{3}$. The power and energy radiated during the post-Hawking burst for $u_{2}<u<u_{3}$ and the late-time burst for $\tilde{u}_{2}<u<\tilde{u}_{3}$ are approximately the same in order of magnitude as

$$
P \simeq \epsilon^{-4 \beta} P_{H} \quad \text { and } \quad E \simeq \frac{1}{48 \pi} \frac{1}{8 M} \epsilon^{-2 \beta}
$$

for the duration $u_{3}-u_{2} \simeq \tilde{u}_{3}-\tilde{u}_{2} \simeq 2 M \epsilon^{2 \beta}$ as discussed in Appendix C. Both of the bursts dominate the transient Hawking radiation in both power and energy. The evolution of radiation is summarized for $\beta=1 / 2$ and $\beta=1$ in Fig. 6.

\section{Instant deceleration limit}

It is interesting to see the limit $\beta \rightarrow \infty$ or $R_{b}-R_{f} \rightarrow 0$ while $1-\left(2 M / R_{f}\right)=\epsilon^{2}$ is fixed in both models $\mathrm{A}$ and $\mathrm{B}$. In this limit, the power becomes stronger and stronger, the time width becomes shorter and shorter, and the energy radiated becomes more and more in both the post-Hawking 
and late-time bursts, while the duration of the dormant stage in between is unchanged. Thus, we can reproduce the last two delta-functional bursts in the null-shell model in Sec. V.

\section{DISCUSSION}

It is important to compare our result with previous results in similar setups. In Refs. [34,35], a timelike-shell model was also used, the end state of which is a static shell with radius slightly larger than $2 M$. However, instead of prescribing the shell dynamics, the function $G(u)$ was assumed directly to satisfy the expected qualitative asymptotic properties and change in a timescale of the order of $M$. Figures 7 and 9 in Refs. [34,35] indicate that the width of the late-time burst is several tens of $M$ and the power is bounded by that of the Hawking radiation $P_{H}$. It was also observed that the width of the burst increases for smaller $\epsilon$. As seen in Sec. VID, these features correspond to our model A with $\beta=1 / 2$. On the other hand, we can argue that the physically natural scenario corresponds to model A with $\beta=1$ from the argument that the shell begins to brake when $R=R_{b}=R_{f}+2 M \epsilon^{2}$ and settles down to $R=R_{f}=$ $2 M /\left(1-\epsilon^{2}\right)$, if there is a unique characteristic scale which controls both the braking and the freeze-in of the shell and that the force onto the shell is vanishingly small near $R=R_{f}$, if $R=R_{f}$ is the radius of equilibrium.

It is interesting to estimate the quantities which appear here using astrophysical values. The transient Hawking radiation lasts for $u_{1}-u_{0} \sim 40\left(M / M_{\odot}\right)\left[44+\ln \left(10^{-19} / \epsilon\right)\right] \mu \mathrm{s}$. The radiation itself carries a power, temperature, and energy:

$$
\begin{gathered}
P \simeq P_{H} \sim 10^{-21}\left(\frac{M}{M_{\odot}}\right)^{-2} \mathrm{erg} / \mathrm{s}, \\
T_{H} \sim 6 \times 10^{-8}\left(\frac{M}{M_{\odot}}\right)^{-1} \mathrm{~K}, \\
E \simeq 4 \times 10^{-26}\left(\frac{M}{M_{\odot}}\right)^{-1}\left[44+\ln \left(\frac{10^{-19}}{\epsilon}\right)\right] \mathrm{erg} .
\end{gathered}
$$

The subsequent "dormant" stage lasts for $\tilde{u}_{1}-u_{3} \sim 6 \times$ $10^{6}\left(M / M_{\odot}\right)\left(\epsilon / 10^{-19}\right)^{-1} \mathrm{yr}$ and is followed by a late-time burst whose details depend on the model.

For model A with $\beta=1 / 2$, the late-time burst lasts for $\tilde{u}_{2}-\tilde{u}_{1} \sim 2 M\left(\ln \epsilon^{-1}\right) \sim 10\left(M / M_{\odot}\right)\left[44+\ln \left(10^{-19} / \epsilon\right)\right] \mu$ s and is characterized by $P \sim P_{H}, T=T_{H}$, and $E \sim E_{H}$. For model A with $\beta=1$, the late-time burst lasts for $\tilde{u}_{2}-\tilde{u}_{1} \sim$ $10^{-24}\left(M / M_{\odot}\right)\left(\epsilon / 10^{-19}\right)\left[44+\ln \left(10^{-19} / \epsilon\right)\right] \mathrm{s}$ and carries power, equivalent temperature $T_{\text {eq }}$, effective temperature $T_{\text {eff }}$, and energy,

$$
\begin{gathered}
P \sim 10^{17}\left(\frac{\epsilon}{10^{-19}}\right)^{-2}\left(\frac{M}{M_{\odot}}\right)^{-2} \mathrm{erg} / \mathrm{s}, \\
k T_{\mathrm{eq}} \sim-100\left(\frac{\epsilon}{10^{-19}}\right)^{-1}\left(\frac{M}{M_{\odot}}\right)^{-1} \mathrm{MeV},
\end{gathered}
$$

$$
k T_{\text {eff }} \sim 0.1\left(\frac{\epsilon}{10^{-19}}\right)^{-1 / 2}\left(\frac{M}{M_{\odot}}\right)^{-1} \mathrm{eV},
$$

$$
E \sim 10^{-7}\left(\frac{\epsilon}{10^{-19}}\right)^{-1}\left(\frac{M}{M_{\odot}}\right)^{-1}\left[44+\ln \left(10^{-19} / \epsilon\right)\right] \mathrm{erg},
$$

where $k T_{\text {eq }}:=\kappa /(2 \pi)$ and $k T_{\text {eff }}:=\left(P /\left(4 \pi R^{2} \sigma_{\mathrm{SB}} / 2\right)\right)^{1 / 4}$ with $\sigma_{\mathrm{SB}}=\pi^{2} k^{4} / 60$ the Stefan-Boltzmann constant, while the post-Hawking burst carries approximately the same power and same equivalent and effective temperatures. For model B with $\beta=1$, these observables for both the first and the second bursts are given by

$$
\begin{aligned}
P & \sim 10^{55}\left(\frac{\epsilon}{10^{-19}}\right)^{-4}\left(\frac{M}{M_{\odot}}\right)^{-2} \mathrm{erg} / \mathrm{s}, \\
k T_{\mathrm{eq}} & \sim-10^{18}\left(\frac{\epsilon}{10^{-19}}\right)^{-2}\left(\frac{M}{M_{\odot}}\right)^{-1} \mathrm{GeV}, \\
k T_{\mathrm{eff}} & \sim 10\left(\frac{\epsilon}{10^{-19}}\right)^{-1}\left(\frac{M}{M_{\odot}}\right)^{-1} \mathrm{MeV} \\
E & \sim 10^{12}\left(\frac{\epsilon}{10^{-19}}\right)^{-2}\left(\frac{M}{M_{\odot}}\right)^{-1} \mathrm{erg},
\end{aligned}
$$

with time widths $u_{3}-u_{2} \simeq \tilde{u}_{3}-\tilde{u}_{2} \simeq 10^{-43}\left(\epsilon / 10^{-19}\right)^{2} \mathrm{~s}$.

Here, we would like to discuss some remaining issues. The first concerns arguments for the "expected" values of our $\epsilon$ parameter. Although $\epsilon \sim \sqrt{\ell_{\mathrm{Pl}} /(2 M)} \simeq 10^{-19}\left(M / M_{\odot}\right)^{-1 / 2}$ is suggested by some semiclassical arguments [10], other scenarios where $\epsilon$ can be much larger or smaller than this value are possible. For example, one might identify the proper length from the surface with the Planck length ${ }^{2}$ (instead of the areal radius). In such a case, $\epsilon$ can be as small as $\epsilon \simeq l_{P} /(4 M) \simeq 10^{-38}\left(M / M_{\odot}\right)^{-1}$ and our results become even more extreme. On the other hand, if we consider a neutron star, we may estimate $\epsilon \sim 0.5$, for which the present formulation is only marginally valid.

We have shown that the duration of the dormant stage is $\sim 4 M / \epsilon$. Physically, the $4 M$ factor is simply the proper time of the shell for a null ray to cross its diameter, when the shell is sufficiently close to $2 M$. The factor $1 / \epsilon$ comes from the redshift factor between the proper time of the almost static shell and the observer time.

The particle production process is characterized by different stages, after what we termed the "standard" collapse phase. This large number of particle production stages is due to the different classes of null-ray pairs that govern quantum particle creation. We can summarize the

\footnotetext{
${ }^{2}$ The authors are grateful to T. Tanaka for pointing out this possibility.
} 
correspondence as follows: braking at $\tau=\tau_{\text {out }}$ and standard collapse at $\tau=\tau_{\text {in }}$ contribute to the post-Hawking burst, the final static phase at $\tau=\tau_{\text {out }}$ and standard collapse at $\tau=\tau_{\text {in }}$ produce the dormant stage, whereas the final static phase at $\tau=\tau_{\text {out }}$ and braking at $\tau=\tau_{\text {in }}$ give the late-time burst.

We have applied the geometrical optics approximation in the entire treatment. This is valid for $s$-waves and for sufficiently high frequencies. On the other hand, the reflection of waves by the shell and the geometry is completely neglected. This implies that if we relax this approximation, we will obtain not only the post-Hawking and late-time bursts but also echoes in particle creation due to the reflections of waves (cf. Refs. [2,3,5]). The details of this process require further calculations.

As we pointed out, we adopted the same set of assumptions for calculating quantum particle creation from a collapsing body as previous works in the literature $[18,19,26]$. It is clearly important to go beyond such restrictions. If one goes beyond the geometrical optics approximation, Hawking radiation appears as a stationary process at the final stage of the collapse to a black hole with various intermediate decaying stages [36]. Quantum loop corrections to the flux of non-Gaussian (self-interacting) theories are not suppressed in comparison with the tree-level contribution in the case of $\lambda \phi^{4}$ theory [36]. This may also modify the properties of the Hawking radiation and perhaps those of the bursts discussed in the current paper. Furthermore, the properties of radiation in the intermediate stage of the collapse may strongly depend on the choice of the initial quantum state.

Finally, we have prescribed the shell dynamics in this paper, but postpone a discussion about the matter content of the shell which enables such an unusual time evolution. We expect that some energy conditions must be violated. The physical significance of such violations is not completely clear. However, we take this opportunity to once more stress that one of the main goals of this work is to look for distinctive features of horizonless objects as a way to strengthen the black hole paradigm.

\section{ACKNOWLEDGMENTS}

The authors are grateful to T. Igata, S. Kinoshita, H. Maeda, A. Matsumura, F. C. Mena, K. i. Nakao, K. Nakashi, J. M. M. Senovilla, and T. Tanaka for helpful comments and fruitful discussions. This work was partially supported by JSPS KAKENHI Grant No. JP26400282 (T. H.). T. H. thanks CENTRA, IST, Lisbon, for hospitality received during this work. The authors are grateful to Yukawa Institute for Theoretical Physics at Kyoto University for hospitality while this work was completed during the YITP-T-18-05 on "Dynamics in Strong Gravity Universe." T. H. was supported by Rikkyo University International Academic Research Exchange. The authors acknowledge financial support provided under the European Union's H2020 ERC Consolidator Grant "Matter and strongfield gravity: New frontiers in Einstein's theory" Grant
Agreement No. MaGRaTh-646597. The authors acknowledge financial support provided by FCT/Portugal through Grant No. PTDC/MAT-APL/30043/2017. This work has received funding from the European Union's Horizon 2020 research and innovation program under the Marie Skłodowska-Curie Grant Agreement No. 690904, and through NSF-XSEDE Award No. PHY-090003. This article is based upon work from COST Action CA16104 "GWverse," supported by COST (European Cooperation in Science and Technology).

\section{APPENDIX A: EXPRESSIONS FOR A TIMELIKE-SHELL MODEL}

The junction condition for the first fundamental form gives

$$
\dot{T}^{2}=1+\dot{R}^{2}, \quad \dot{t}^{2}=\frac{1}{1-\frac{2 M}{R}}\left(1+\frac{\dot{R}^{2}}{1-\frac{2 M}{R}}\right) .
$$

The relation between the null coordinates and the proper time of the shell is given by

$$
\dot{U}=\sqrt{1+\dot{R}^{2}}-\dot{R}, \quad \dot{V}=\sqrt{1+\dot{R}^{2}}+\dot{R},
$$

and

$\dot{u}=\frac{\sqrt{1-\frac{2 M}{R}+\dot{R}^{2}}-\dot{R}}{1-\frac{2 M}{R}}, \quad \dot{v}=\frac{\sqrt{1-\frac{2 M}{R}+\dot{R}^{2}}+\dot{R}}{1-\frac{2 M}{R}}$

From Eqs. (A2) and (A3), we can write down the explicit expression for $A$ and $B$ in terms of $R$ as follows:

$$
\begin{aligned}
& A=\frac{\left(1-\frac{2 M}{R}\right)\left(\sqrt{1+\dot{R}^{2}}-\dot{R}\right)}{\sqrt{1-\frac{2 M}{R}+\dot{R}^{2}}-\dot{R}}, \\
& B=\frac{\left(1-\frac{2 M}{R}\right)\left(\sqrt{1+\dot{R}^{2}}+\dot{R}\right)}{\sqrt{1-\frac{2 M}{R}+\dot{R}^{2}}+\dot{R}} .
\end{aligned}
$$

From Eqs. (A3) and (A4), we can write down the expression for $C$ and $D$ in terms of $R$ as follows:

$$
\begin{aligned}
C= & -\frac{\ddot{R}\left(1-\frac{2 M}{R}\right)}{\sqrt{1-\frac{2 M}{R}+\dot{R}^{2}}-\dot{R}}\left[\frac{1}{\sqrt{1-\frac{2 M}{R}+\dot{R}^{2}}}-\frac{1}{\sqrt{1+\dot{R}^{2}}}\right] \\
& -\frac{M \dot{R}}{R^{2} \sqrt{1-\frac{2 M}{R}+\dot{R}^{2}}},
\end{aligned}
$$




$$
\begin{aligned}
D= & \frac{\ddot{R}\left(1-\frac{2 M}{R}\right)}{\sqrt{1-\frac{2 M}{R}+\dot{R}^{2}}+\dot{R}}\left[\frac{1}{\sqrt{1-\frac{2 M}{R}+\dot{R}^{2}}}-\frac{1}{\sqrt{1+\dot{R}^{2}}}\right] \\
& -\frac{M \dot{R}}{R^{2} \sqrt{1-\frac{2 M}{R}+\dot{R}^{2}}} .
\end{aligned}
$$

\section{APPENDIX B: EXPRESSIONS FOR A TIMELIKE-SHELL MODEL IN DIFFERENT REGIMES}

To estimate the functions $A, B, C$, and $D$, we are interested in the following phases: $0 .(R \gg 2 M$ and $|\dot{R}| \ll 1), 1$, 2 . $\left(1-\frac{2 M}{R} \ll 1\right.$ and $\left.1-\frac{2 M}{R} \ll \dot{R}^{2}\right), \quad 3$. $\quad\left(1-\frac{2 M}{R} \ll 1\right.$ and $\left.1-\frac{2 M}{R} \ll \dot{R}^{2}\right)$, and 4 . $(R=$ const). Let us consider these cases separately. We assume $\dot{R}<0$ in the following.

0. $R \gg 2 M$ and $|\dot{R}| \ll 1$

We find

$A \simeq 1, \quad B \simeq 1, \quad C \simeq-\frac{M}{R}\left(\ddot{R}-\frac{|\dot{R}|}{R}\right)$,

$D \simeq \frac{M}{R}\left(\ddot{R}+\frac{|\dot{R}|}{R}\right)$.

From Eqs. (A2) and (A3), we obtain

$$
\begin{array}{rlrl}
U \simeq \tau+\text { const }, & V \simeq \tau+\text { const }, \\
u \simeq \tau+\text { const }, & & & \simeq \tau+\text { const } .
\end{array}
$$

1. and $2.1-\frac{2 M}{R} \ll 1$ and $1-\frac{2 M}{R} \ll \dot{R}^{2}$

In this regime, we have

$$
\begin{aligned}
& A \simeq\left(1-\frac{2 M}{R}\right) \frac{\sqrt{1+\dot{R}^{2}}+|\dot{R}|}{2|\dot{R}|}, \\
& B \simeq 2|\dot{R}|\left(\sqrt{1+\dot{R}^{2}}-|\dot{R}|\right), \\
& C \simeq-\frac{\ddot{R}}{2 \dot{R}^{2}}\left(1-\frac{2 M}{R}\right)\left[1-\frac{|\dot{R}|}{\sqrt{1+\dot{R}^{2}}}\right]+\frac{1}{4 M}, \\
& D \simeq 2 \ddot{R}\left[1-\frac{|\dot{R}|}{\sqrt{1+\dot{R}^{2}}}\right]+\frac{1}{4 M} .
\end{aligned}
$$

In this case, from Eqs. (A3), $u$ and $v$ are given by

$u \simeq-4 M \ln \left[\frac{R}{2 M}-1\right]+$ const, $\quad v \simeq-\frac{1}{2} \int \frac{d R}{\dot{R}^{2}}$.

If we further assume $\dot{R}=O(1)$ and $\ddot{R}=O\left((2 M)^{-1}\right)$ corresponding to phase 1 , we obtain

$$
\begin{aligned}
& A=O\left(\left(1-\frac{2 M}{R}\right)\right), \quad B=O(1), \\
& C \simeq \frac{1}{4 M}, \quad D=O\left((2 M)^{-1}\right) .
\end{aligned}
$$

In this case, Eqs. (A2) imply

$$
U \sim \tau+\text { const, } \quad V \sim \tau+\text { const. }
$$

3. $1-\frac{2 M}{R} \ll 1$ and $1-\frac{2 M}{R} \gg \dot{R}^{2}$

In this regime, we have

$$
\begin{array}{ll}
A \simeq \sqrt{1-\frac{2 M}{R}}, \quad B \simeq \sqrt{1-\frac{2 M}{R}}, \quad C \simeq-\ddot{R}+\frac{|\dot{R}|}{4 M \sqrt{1-\frac{2 M}{R}}}, \\
D \simeq \ddot{R}+\frac{|\dot{R}|}{4 M \sqrt{1-\frac{2 M}{R}}} .
\end{array}
$$

In this case, from Eqs. (A2) and (A3), we obtain

$U \simeq \tau+$ const,$\quad V \simeq \tau+$ const,$\quad u \simeq \int \frac{d \tau}{\sqrt{1-\frac{2 M}{R}}}$,

$v \simeq \int \frac{d \tau}{\sqrt{1-\frac{2 M}{R}}}$

4. $R=$ const

In this regime, we have

$$
A=B=\sqrt{1-\frac{2 M}{R}}, \quad C=D=0,
$$

and

$U=\tau+$ const,$\quad V=\tau+$ const,$\quad u=\frac{\tau}{\sqrt{1-\frac{2 M}{R}}}+$ const,

$v=\frac{\tau}{\sqrt{1-\frac{2 M}{R}}}+$ const.

\section{APPENDIX C: TIME INTERVALS}

Since $R=4 M$ at $u=u_{0}$ and $R=R_{b}=R_{f}+2 M \epsilon^{2 \beta}$ at $u=u_{1}$, Eq. (B7) implies

$$
u_{1}-u_{0} \simeq\left\{\begin{array}{cc}
4 M \beta \ln \epsilon^{-2} & (0<\beta<1) \\
4 M \ln \epsilon^{-2} & (\beta \geq 1)
\end{array} .\right.
$$

Equations (A2) and (A3) imply that the intervals in terms of $u$ are given as follows:

$$
\begin{aligned}
& \tilde{u}_{2}-u_{2} \simeq \epsilon^{-1}\left(\tilde{\tau}_{2}-\tau_{2}\right) \simeq \frac{4 M}{\epsilon}, \\
& \tilde{u}_{3}-u_{3} \simeq \epsilon^{-1}\left(\tilde{\tau}_{3}-\tau_{3}\right) \simeq \frac{4 M}{\epsilon},
\end{aligned}
$$

where we have used $\tilde{\tau}_{2}-\tau_{2} \simeq \tilde{\tau}_{3}-\tau_{3} \simeq 4 M$. The above relations do not depend on the details of the model. 


\section{Model A: Exponentially slowed-down model}

First, we estimate $\tau_{2}$. Assuming $R-R_{f} \propto e^{-\sigma \tau}$ for $\tau_{1}<\tau<\tau_{3}^{\prime}$ with $\sigma$ given by Eq. (6.8), we find

$1-\frac{2 M}{R} \simeq\left(1-\frac{2 M}{R_{f}}\right)+\left(\frac{2 M}{R_{f}}-\frac{2 M}{R}\right) \simeq \epsilon^{2}+\epsilon^{2 \beta} e^{-\sigma\left(\tau-\tau_{1}\right)}$

for $\quad R_{b}-R_{f}=2 M \epsilon^{2 \beta} \quad$ with $\quad \beta \geq 1 / 2$. Noting $|\dot{R}|=$ $\left|\dot{R}_{b}\right| e^{-\sigma\left(\tau-\tau_{1}\right)}$ and $\left|\dot{R}_{b}\right|=O(1)$, Eq. (6.2) at $\tau=\tau_{2}$ implies

$$
\tau_{2}-\tau_{1} \simeq \sigma^{-1}\left(\ln \epsilon^{-1}\right) \quad \text { and } \quad 1-\frac{2 M}{R_{2}} \simeq \epsilon^{2} .
$$

Then, we can derive

$$
\begin{aligned}
& u_{2}-u_{1} \simeq\left\{\begin{array}{cc}
4 M(1-\beta) \ln \epsilon^{-2} & (1 / 2 \leq \beta<1) \\
4 M & (\beta=1) \\
4 M \epsilon^{2(\beta-1)} & (\beta>1)
\end{array},\right. \\
& u_{2}-u_{0} \simeq 4 M \ln \epsilon^{-2} .
\end{aligned}
$$

Equations (A2) and (A3) imply that the intervals in terms of $u$ are given as follows:

$$
\begin{aligned}
\tilde{u}_{2}-\tilde{u}_{1} & \simeq \epsilon^{-1}\left(\tilde{\tau}_{2}-\tilde{\tau}_{1}\right) \sim \epsilon^{-1}\left(\tau_{2}-\tau_{1}\right) \sim \epsilon^{-1}\left(\ln \epsilon^{-1}\right) \sigma^{-1} \\
& \sim 2 M \epsilon^{2 \beta-1}\left(\ln \epsilon^{-1}\right),
\end{aligned}
$$

where we have used $\sigma \simeq 1 /\left(R_{b}-R_{f}\right)$. Additionally assuming $\tau_{3}-\tau_{2} \simeq \sigma^{-1}$, we can find

$$
u_{3}-u_{2} \simeq \tilde{u}_{3}-\tilde{u}_{2} \simeq \epsilon^{-1} \sigma^{-1} \simeq 2 M \epsilon^{2 \beta-1} .
$$

\section{Model B: Constant-deceleration model}

In this model, we find

$$
1-\frac{2 M}{R} \simeq \epsilon^{2}+\frac{a}{4 M}\left(\tau_{3}-\tau\right)^{2}
$$

for $\tau_{1}<\tau<\tau_{3}$ with $a$ given by Eq. (6.16). Noting $|\dot{R}|=$ $a\left(\tau_{3}-\tau\right)$ and $\left|\dot{R}_{b}\right|=O(1), \tau_{2}$ is estimated as

$$
\tau_{3}-\tau_{2}=\frac{\epsilon}{\sqrt{a\left(a-\frac{1}{4 M}\right)}},
$$

while $\tau_{3}$ is estimated as $\tau_{3}-\tau_{1}=\left|\dot{R}_{b}\right| a^{-1}$. Then, we can derive

$$
\begin{aligned}
& u_{2}-u_{1} \simeq\left\{\begin{array}{cc}
4 M(1-\beta) \ln \epsilon^{-2} & (0<\beta<1) \\
4 M & (\beta=1) \\
4 M \epsilon^{2(\beta-1)} & (\beta>1)
\end{array},\right. \\
& u_{2}-u_{0} \simeq 4 M \ln \epsilon^{-2} .
\end{aligned}
$$

The expressions for $u_{3}-u_{2}, \tilde{u}_{3}-\tilde{u}_{1}$, and $\tilde{u}_{3}-\tilde{u}_{2}$ are given by

$u_{3}-u_{2} \simeq \epsilon^{-1}\left(\tau_{3}-\tau_{2}\right) \simeq a^{-1} \simeq 4 M \epsilon^{2 \beta}$,

$\tilde{u}_{3}-\tilde{u}_{1} \simeq \epsilon^{-1}\left(\tau_{3}-\tau_{1}\right) \simeq\left|\dot{R}_{b}\right| \epsilon^{-1} a^{-1} \simeq 4 M \epsilon^{2 \beta-1}$,

$\tilde{u}_{3}-\tilde{u}_{2} \simeq \epsilon^{-1}\left(\tau_{3}-\tau_{2}\right) \simeq a^{-1} \simeq 4 M \epsilon^{2 \beta}$,

where we have assumed $a \gg 1 /(4 M)$ and $a \simeq 1 /$ $\left[2\left(R_{b}-R_{f}\right)\right]=1 /\left(4 M \epsilon^{2 \beta}\right)$.

\section{APPENDIX D: DETAILED ANALYSIS OF THE TEMPORAL CHANGE OF RADIATION}

We divide the observer's time to eight intervals: $u<u_{0}$, $u_{0}<u<u_{1}, \quad u_{1}<u<u_{2}, \quad u_{2}<u<u_{3}, \quad u_{3}<u<\tilde{u}_{1}$, $\tilde{u}_{1}<u<\tilde{u}_{2}, \tilde{u}_{2}<\tilde{u}_{3}$, and $\tilde{u}_{3}<u$. Since the first two are identical to those in the standard-collapse phase, discussed in Sec. IV, we concentrate on the last six. For each interval, the classes of null-ray pairs are fixed and we can obtain the expressions for the functions $G^{\prime}(u)$ and $\kappa(u)$ by combining the expressions for $A, B, C$, and $D$ given in Appendix B through the formula (4.3).

(i) $u_{1}<u<u_{2}$ : We discuss this stage in Sec. VIB. There are null-ray pairs of classes $(2,1)$ and $(2,0)$. There is no pair of class $(2,2)$ because the duration of phase $2, \tau_{2}-\tau_{1}$, is much shorter than the time for return travel, which is approximately $4 M$.

(ii) $u_{2}<u<u_{3}$ : We also discuss this stage in Sec. VI B. We have null-ray pairs of classes $(3,0)$ and $(3,1)$. There is no pair of class $(3,2)$ or $(3,3)$ because we assume that $\tau_{3}-\tau_{2}$ is much shorter than $4 M$.

(iii) $u_{3}<u<\tilde{u}_{1}$ : All null-ray pairs are of class $(4,0)$ or $(4,1)$. We can find $\kappa=O\left(\epsilon(2 M)^{-1}\right)$, which is the contribution from $\tau=\tau_{\text {in }}$ in phases 0 and 1, irrespectively of the model details. This corresponds to the long dormant stage.

(iv) $\tilde{u}_{1}<u<\tilde{u}_{2}$ : We discuss this stage in Sec. VIC. The null-ray pairs are of class $(4,2)$.

(v) $\tilde{u}_{2}<u<\tilde{u}_{3}$ : We also discuss this stage in Sec. VI C. We have null-ray pairs of class $(4,3)$.

(vi) $\tilde{u}_{3}<u$ : We have null-ray pairs of class $(4,4)$. For this class, we have just $G^{\prime}(u)=1$ and $\kappa(u)=0$. The radiation completely vanishes at $u=\tilde{u}_{3}$ and thereafter no radiation is emitted forever. 
[1] M. A. Abramowicz, W. Kluzniak, and J. P. Lasota, No observational proof of the black hole event-horizon, Astron. Astrophys. 396, L31 (2002).

[2] V. Cardoso and P. Pani, Tests for the existence of horizons through gravitational wave echoes, Nat. Astron. 1, 586 (2017).

[3] V. Cardoso, E. Franzin, and P. Pani, Is the GravitationalWave Ringdown a Probe of the Event Horizon?, Phys. Rev. Lett. 116, 171101 (2016); Erratum 117, 089902(E) (2016).

[4] K. i. Nakao, C. M. Yoo, and T. Harada, On gravastar formation: What can be the evidence of a black hole?, arXiv: 1809.00124.

[5] V. Cardoso and P. Pani, The observational evidence for horizons: from echoes to precision gravitational-wave physics, arXiv:1707.03021.

[6] V. Cardoso, E. Franzin, A. Maselli, P. Pani, and G. Raposo, Testing strong-field gravity with tidal Love numbers, Phys. Rev. D 95, 084014 (2017); Publisher's Note 95, 089901(A) (2017).

[7] A. Maselli, P. Pani, V. Cardoso, T. Abdelsalhin, L. Gualtieri, and V. Ferrari, Probing Planckian Corrections at the Horizon Scale with LISA Binaries, Phys. Rev. Lett. 120, 081101 (2018).

[8] R. Shaikh, P. Kocherlakota, R. Narayan, and P. S. Joshi, Shadows of spherically symmetric black holes and naked singularities, Mon. Not. R. Astron. Soc. 482, 52 (2019).

[9] P. V. P. Cunha, C. A. R. Herdeiro, and M. J. Rodriguez, Does the black hole shadow probe the event horizon geometry?, Phys. Rev. D 97, 084020 (2018).

[10] P. O. Mazur and E. Mottola, Gravitational vacuum condensate stars, Proc. Natl. Acad. Sci. U.S.A. 101, 9545 (2004).

[11] M. Visser and D. L. Wiltshire, Stable gravastars: An Alternative to black holes?, Classical Quantum Gravity 21, 1135 (2004).

[12] M. Visser, C. Barcelo, S. Liberati, and S. Sonego, Small, dark, and heavy: But is it a black hole?, Proc. Sci., BHGRS2008 (2008) 010 [arXiv:0902.0346].

[13] C. Berthiere, D. Sarkar, and S. N. Solodukhin, The fate of black hole horizons in semiclassical gravity, Phys. Lett. B 786, 21 (2018).

[14] N. Sakai, H. Saida, and T. Tamaki, Gravastar Shadows, Phys. Rev. D 90, 104013 (2014).

[15] T. Kubo and N. Sakai, Gravitational lensing by gravastars, Phys. Rev. D 93, 084051 (2016).

[16] C. B. M. H. Chirenti and L. Rezzolla, How to tell a gravastar from a black hole, Classical Quantum Gravity 24, 4191 (2007).

[17] C. Chirenti and L. Rezzolla, Did GW150914 produce a rotating gravastar?, Phys. Rev. D 94, 084016 (2016).
[18] S. W. Hawking, Black hole explosions, Nature (London) 248, 30 (1974).

[19] S. W. Hawking, Particle Creation by Black Holes, Commun. Math. Phys. 43, 199 (1975); Erratum 46, 206(E) (1976).

[20] N. D. Birrell and P. C. W. Davies, Quantum Fields in Curved Space (Cambridge University Press, Cambridge, England, 1984).

[21] C. Barcelo, S. Liberati, S. Sonego, and M. Visser, Hawkinglike radiation from evolving black holes and compact horizonless objects, J. High Energy Phys. 02 (2011) 003.

[22] C. Barcelo, S. Liberati, S. Sonego, and M. Visser, Fate of gravitational collapse in semiclassical gravity, Phys. Rev. D 77, 044032 (2008).

[23] S. Kinoshita and N. Tanahashi, Hawking temperature for near-equilibrium black holes, Phys. Rev. D 85, 024050 (2012).

[24] P. Chen, W. G. Unruh, C. H. Wu, and D. H. Yeom, PreHawking radiation cannot prevent the formation of apparent horizon, Phys. Rev. D 97, 064045 (2018).

[25] W. G. Unruh, Prehawking radiation, arXiv:1802.09107.

[26] L. H. Ford and L. Parker, Creation of Particles by Singularities in Asymptotically Flat Space-Times, Phys. Rev. D 17, 1485 (1978).

[27] W. A. Hiscock, L. G. Williams, and D. M. Eardley, Creation Of Particles By Shell Focusing Singularities, Phys. Rev. D 26, 751 (1982).

[28] S. Barve, T. P. Singh, C. Vaz, and L. Witten, Particle creation in the marginally bound, selfsimilar collapse of inhomogeneous dust, Nucl. Phys. B532, 361 (1998).

[29] S. Barve, T. P. Singh, C. Vaz, and L. Witten, The Quantum stress tensor in selfsimilar spherical dust collapse, Phys. Rev. D 58, 104018 (1998).

[30] C. Vaz and L. Witten, On the spectrum of the radiation from a naked singularity, Phys. Lett. B 442, 90 (1998).

[31] T. Harada, H. Iguchi, and K. i. Nakao, Naked singularity explosion, Phys. Rev. D 61, 101502 (2000).

[32] T. Harada, H. Iguchi, and K. i. Nakao, Power, energy, and spectrum of a naked singularity explosion, Phys. Rev. D 62, 084037 (2000).

[33] T. Harada, H. Iguchi, and K. i. Nakao, Physical processes in naked singularity formation, Prog. Theor. Phys. 107, 449 (2002).

[34] A. Paranjape and T. Padmanabhan, Radiation from collapsing shells, semiclassical backreaction and black hole formation, Phys. Rev. D 80, 044011 (2009).

[35] K. Banerjee and A. Paranjape, Semiclassical environment of collapsing shells, Phys. Rev. D 80, 124006 (2009).

[36] E. T. Akhmedov, H. Godazgar, and F. K. Popov, Hawking radiation and secularly growing loop corrections, Phys. Rev. D 93, 024029 (2016). 